\title{
A DYNAMICAL MEANING OF FRACTAL DIMENSION
}

BY

\author{
STEVE PELIKAN
}

\begin{abstract}
When two attractors of a dynamical system have a common basin boundary $B$, small changes in initial conditions which lie near $B$ can result in radically different long-term behavior of the trajectory. A quantitative description of this phenomenon is obtained in terms of the fractal dimension of the basin boundary $B$.
\end{abstract}

Introduction. This paper contains theorems concerning the fractal dimension of certain invariant sets of dynamical systems. Rather than computing the dimension of invariant sets, we describe a phenomenon observed in a variety of systems which admits a quantitative description in terms of the fractal dimension of an invariant set.

The sets of interest form a boundary between the basins of attraction of two attractors. The map $f: \mathbf{R} \rightarrow \mathbf{R}$ shown in Figure 1 provides a good example. The map $f$ has two attracting fixed points, $A$ and $B$. Almost every initial condition $x_{0} \in \mathbf{R}$ tends to one of these two points under the action of $f$. The exceptions to this rule are the points of a Cantor set $\Lambda$ contained in $[0,1]$.

In $\$ 2$ we consider the following question. Suppose $x$ and $y$ are chosen at random from $[0,1]$ subject only to the condition $|x-y|<\varepsilon$. What is the probability $p_{\varepsilon}$ that $x$ and $y$ tend to different attractors? If $f$ describes a physical system this question can be phrased as: Suppose there is a uniform $\varepsilon$-error in determining the initial conditions of the system. What is the probability that our prediction of the long-term behavior of the system will be incorrect? We show (Theorem 2) that $p_{\varepsilon}$ tends to zero like $\varepsilon^{1-d}$ as $\varepsilon$ goes to zero, where $d$ is the Hausdorff dimension of $\Lambda$. Thus, when $d>0$ (and espcially when $d$ is near 1) it may be very difficult to predict the long-term behavior of the system correctly, even though the long-term behavior of almost every trajectory is very simple.

This phenomenon, called "final state sensitivity", was studied numerically by the authors of [1]. They actually discuss two measures of the degree of sensitivity. The numerical experiments they describe are concerned with measuring the quantity $p_{\varepsilon}$. In addition, they consider $q_{\varepsilon}$, which is the measure of the set of $x$ for which there exists a $y$ within $\varepsilon$ of $x$ so that $x$ and $y$ tend to different attractors. In [1] it is conjectured that $q_{\varepsilon} \sim \varepsilon^{1-d}$, where $d$ is the dimension of $\Lambda$.

The motivation for studying $p_{\varepsilon}$ (rather than $q_{\varepsilon}$ ) is primarily that it represents a quantity with a more natural interpretation. Returning to the interpretation given

Received by the editors July 11, 1984 and, in revised form, March 1, 1985.

1980 Mathematics Subject Classification. Primary 58F13. 


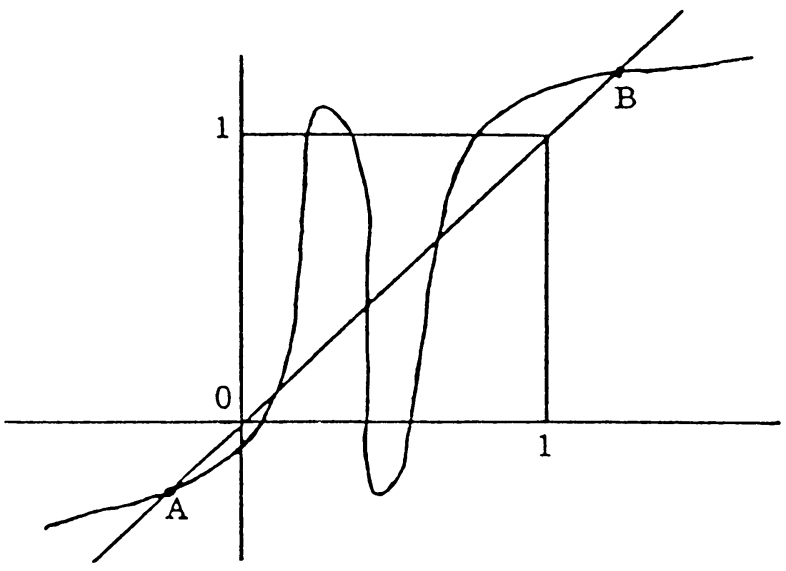

FIGURE 1

above, in which $f$ represents a physical system, $p_{\varepsilon}$ is the probability of making an incorrect prediction of the long-term behavior while $q_{\varepsilon}$ is the probability of being capable of making an incorrect prediction in the presence of $\varepsilon$-uncertainty about the initial condition. In addition, as the numerical techniques of [1] show, $p_{\varepsilon}$ can be computed quite easily, while $q_{\varepsilon}$ cannot.

$\$ 3$ describes two examples of dynamical systems in two dimensions for which theorems analogous to Theorem 2 are true. These are the "linear" horseshoe and certain rational maps of the Riemann sphere.

In $\S 1$ we show that, for invariant sets such as $\Lambda$, the two most frequently used definitions of fractal dimension (i.e., the Hausdorff and capacity dimensions) are equal. The utility of this result comes from the fact that the capacity dimension is much easier to compute numerically than the Hausdorff dimension.

Much of this work was done while the author was a postdoctoral member of the Institute for Mathematics and its Applications at the University of Minnesota. He thanks Drs. Weinberger and Sell and the staff of the Institute for their hospitality. He also thanks Dr. Mary Rees for many interesting conversations concerning these and related matters.

I. Fractal dimensions. Here $\Lambda \subset[0,1]$ is a compact set. The Hausdorff dimension of $\Lambda$, denoted $\operatorname{HD}(\Lambda)$, is defined as follows:

For $\alpha \in \mathbf{R}$, let

$$
h(\alpha)=\lim _{\varepsilon \rightarrow 0^{+}} \inf _{\|\mathscr{A}\|<\varepsilon} \sum_{\mathscr{A}}\left|A_{i}\right|^{\alpha},
$$

where $|A|=$ diameter of $A, \mathscr{A}$ denotes a finite open cover of $\Lambda$, and $\|\mathscr{A}\|=$ $\max _{A_{i} \in \mathscr{A}}\left|A_{i}\right|$.

One shows (see [2]) that there is a number $\alpha^{*}$ so that $h(\alpha)=0$ if $\alpha>\alpha^{*}$ and $h(\alpha)+\infty$ if $\alpha<\alpha^{*}$. The Hausdorff dimension of $\Lambda$ is $\operatorname{HD}(\Lambda)=\alpha^{*}$.

A second notion of the fractal dimension is the capacity dimension. This is defined by

$$
\operatorname{cap}(\Lambda)=\lim _{\varepsilon \rightarrow 0^{+}} \frac{\log N(\varepsilon)}{-\log \varepsilon} \quad \text { (when the limit exists), }
$$


where $N(\varepsilon)=\min _{\|\mathscr{A}\| \leqslant \varepsilon} \operatorname{card}(\mathscr{Q})$ is the minimum number of sets with diameter $\varepsilon$ needed to cover $\Lambda$.

It is easy to see that $\operatorname{HD}(\Lambda) \leqslant \operatorname{cap}(\Lambda)$, and equally easy to give examples where this inequality is strict, for example, $\Lambda=\{0\} \cup\{1 / n\}_{n=1}^{\infty}$. There are also examples where $\operatorname{cap}(\Lambda)$ is not defined.

Our purpose here is to show that if $\Lambda$ is invariant under an expanding map (that is, satisfies some (nonlinear) self-similarity law), then $\operatorname{cap}(\Lambda)=\operatorname{HD}(\Lambda)$. To be specific,

THEOREM 1. Suppose that $\Lambda$ is invariant under a map $f:[0,1] \rightarrow \mathbf{R}$, where $f$ is $C^{1+\alpha}$ for some $\alpha>0$, and expanding on $f^{-1}([0,1])$. Then cap $(\Lambda)=\operatorname{HD}(\Lambda)$. (Expanding means $\left|f^{\prime}(x)\right| \geqslant \lambda>1$ for some number $\lambda$.)

We denote by $\left\{C_{j}^{n}\right\}$ the components of $f^{-n}([0,1])$. These sets form a natural cover of $\Lambda$. The proof of Theorem 1 depends on the following lemma, which says that the $\left\{C_{j}^{n}\right\}$ 's approximate $\Lambda$ so well that they can be used to compute the Hausdorff dimension of $\Lambda$.

LeMma 1. Let $d>\operatorname{HD}(\Lambda)$. Then

$$
\lim _{n} \sum_{j}\left|C_{j}^{n}\right|^{d}=0
$$

A second, well-known, fact will be required concerning expanding functions $f$ :

Lemma 2. There exists $K \geqslant 1$ independent of $n$ such that

$$
\left|\frac{(d / d x) f^{n}(x)}{(d / d x) f^{n}(y)}\right| \leqslant K
$$

whenever $x, y$ lie in the same set $C_{j}^{n}$.

Proof of Theorem 1. Let $d>\operatorname{HD}(\Lambda)$. Using Lemma 1 , select $N$ so that $n>N$ implies that $\sum_{j}\left|C_{j}^{n}\right|^{d}<K^{-d}$, where $K$ is the constant in Lemma 2. Let $\varepsilon_{0}=$ $\min _{j \neq k} \operatorname{dist}\left(C_{j}^{n}, C_{k}^{n}\right)$. For $\varepsilon<\varepsilon_{0}$, denote by $\mathscr{D}_{\varepsilon}=\left\{D_{1}\right\}$ a cover of $\Lambda$ by $N(\varepsilon)$ sets of diameter $\varepsilon$. The collection $\left\{f^{n}\left(D_{i} \cap C_{j}^{n}\right)\right\}$ is then a cover of $\Lambda$ by sets with diameters in the interval $\left[\varepsilon K^{-1}\left|C_{j}^{n}\right|^{-1}, \varepsilon K\left|C_{j}^{n}\right|^{-1}\right]$, and so

$$
\operatorname{card}\left\{D_{i} \in \mathscr{D}_{\varepsilon}: D_{i} \cap C_{j}^{n} \neq \varnothing\right\} \leqslant N\left(\varepsilon K^{-1}\left|C_{j}^{n}\right|^{-1}\right)
$$

Summing (1) over $j$ we obtain

$$
N(\varepsilon) \leqslant \sum_{j} N\left(\varepsilon K^{-1}\left|C_{j}^{n}\right|^{-1}\right) .
$$

Define $\phi(\varepsilon)$ by $N(\varepsilon)=\varepsilon^{-d} \phi(\varepsilon)$. Substituting this expression for $N(\varepsilon)$ in (2) yields

$$
\phi(\varepsilon) \leqslant K^{d} \sum_{j}\left|C_{j}^{n}\right|^{d} \phi\left(\varepsilon K^{-1}\left|C_{j}^{n}\right|^{-1}\right) .
$$

This expresses a bound for $\phi(\varepsilon)$ as a convex combination of the values of $\phi$ at larger arguments, and so $\phi(\varepsilon)$ is bounded. Consequently,

$$
\varlimsup_{\varepsilon \rightarrow 0} \frac{\log N(\varepsilon)}{-\log \varepsilon} \leqslant d \quad \text { for each } d>\operatorname{HD}(\Lambda),
$$

and so $\operatorname{HD}(\Lambda)=\operatorname{cap}(\Lambda)$. 
Proof of Lemma 1. First, note that it suffices to prove that $\lim _{n} \sum_{j}\left|C_{j}^{n}\right|^{d}$ is bounded for every $d>\operatorname{HD}(\Lambda)$ since if $\sum_{j}\left|C_{j}^{n}\right|^{d-\varepsilon} \leqslant M$ is bounded then

$$
\sum_{j}\left|C_{j}^{n}\right|^{d}=\sum\left|C_{j}^{n}\right|^{d-\varepsilon}\left|C_{j}^{n}\right|^{\varepsilon} \leqslant M \max _{j}\left|C_{j}^{n}\right|^{\varepsilon} \text {. }
$$

But $\max _{j}\left|C_{j}^{n}\right|<\lambda^{-n}$ because $f$ is expanding.

For $d>\operatorname{HD}(\Lambda)$, define

$$
a_{d}(\varepsilon)=\inf \left\{\sum\left|A_{i}\right|^{d}:\left\{A_{i}\right\} \text { is a cover of } \Lambda \text { and }\left|A_{i}\right| \geqslant \varepsilon\right\} \text {. }
$$

Then $a_{d}(\varepsilon)$ is a nondecreasing function of $\varepsilon$ and $a_{d}(\varepsilon) \rightarrow 0$ as $\varepsilon \rightarrow 0$. For each $\varepsilon>0$ there is a cover of $\Lambda, \mathscr{A}_{\varepsilon}$, with $\left|A_{i}\right| \geqslant \varepsilon$ and $\sum_{\mathscr{A}_{i}}\left|A_{i}\right|^{d}<2 a_{d}(\varepsilon)$. We then have that $\left\|\mathscr{A}_{\varepsilon}\right\|^{d}=\max \left|A_{i}\right|^{d}<2 a_{d}(\varepsilon)$ and so $\left\|\mathscr{A}_{\varepsilon}\right\|<\left[2 a_{d}(\varepsilon)\right]^{1 / d}$.

For each $n$, find $\varepsilon_{n}$ so that

$$
\left\|\mathscr{A}_{\varepsilon_{n}}\right\|<\frac{1}{2} \min _{j \neq k} \operatorname{dist}\left(C_{j}^{n}, C_{k}^{n}\right) \text {. }
$$

Then, for each $A_{i} \in \mathscr{A}_{\varepsilon_{n}}$, there is a unique $C_{j}^{n}$ with $A_{i} \cap C_{j}^{n} \neq \varnothing$. We extend $f^{n} \mid C_{j}^{n}$ linearly with slope $\left|C_{j}^{n}\right|^{-1}$. Having done this, $f^{n}\left(A_{i}\right)$ is a well-defined set for each $A_{i}$ which has nonempty intersection with $C_{j}^{n}$. In fact

$$
\left\{f^{n}\left(A_{i}\right): A_{i} \cap C_{j}^{n} \neq \varnothing\right\} \text { is a cover of } \Lambda
$$

by sets having diameters at least $\varepsilon_{n} K^{-1}\left|C_{j}^{n}\right|^{-1}$. Consequently,

$$
\sum_{A_{i} \cap C_{j}^{n} \neq \varnothing}\left|A_{i}\right|^{d} K^{d}\left|C_{j}^{n}\right|^{-d} \geqslant a_{d}\left(\varepsilon_{n} K^{-1}\left|C_{j}^{n}\right|^{-1}\right),
$$

and so

$$
\begin{aligned}
\sum_{i}\left|A_{i}\right|^{d} & =\sum_{j} \sum_{A_{i} \cap C_{j}^{n} \neq \varnothing}\left|A_{i}\right|^{d} \\
& \geqslant \sum_{j} K^{-d}\left|C_{j}^{n}\right|^{d} a_{d}\left(\varepsilon_{n} K^{-1}\left|C_{j}^{n}\right|^{-1}\right) .
\end{aligned}
$$

Now $a_{d}\left(\varepsilon_{n} K^{-1}\left|C_{j}^{n}\right|^{-1}\right) \geqslant a_{d}\left(\varepsilon_{n} K^{-1} \lambda^{n}\right)$ for each $j$, where $\lambda=\min _{x \in f^{-1}[0,1]}\left|f^{\prime}(x)\right|$. Then (4) becomes

$$
2 a_{d}\left(\varepsilon_{n}\right) \geqslant a_{d}\left(\varepsilon_{n} K^{-1} \lambda^{n}\right) K^{-d} \sum_{j}\left|C_{j}^{n}\right|^{d},
$$

or for $n$ such that $\lambda^{n}>K$,

$$
2 K^{d}>2 K^{d} \frac{a_{d}\left(\varepsilon_{n}\right)}{a_{d}\left(\varepsilon_{n} K^{-1} \lambda^{n}\right)} \geqslant \sum_{j}\left|C_{j}^{n}\right|^{d},
$$

by the monotonicity of $a_{d}(\varepsilon)$. This implies $\lim _{n} \sum_{j}\left|C_{j}^{k}\right|^{d}$ is finite.

II. Final state sensitivity. We begin by considering maps $f: \mathbf{R} \rightarrow \mathbf{R}$ which have the essential features of the $f$ shown in Figure 1. We assume

(i) $f$ is $C^{1+\alpha}$,

(ii) $\left|f^{\prime}(x)\right|>1$ if $x$ and $f(x)$ are in $[0,1]$,

(iii) $[0,1] \subset$ interior $(f([0,1]))$ and $f^{-1}[0,1] \subset[0,1]$,

(iv) $f$ has two attractors, one attracting each point in $(1, \infty)$, the other attracting each point in $(-\infty, 0)$. 
We wish to consider the case where the basin boundary of the attractors is a Cantor set. Thus we assume the $f \mid[0,1]$ has at least two laps. (That is, $f^{-1}([0,1])$ consists of at least two intervals.) Let $r$ denote the number of laps of $f$. In case $r=1$, the basin boundary is simply a point and Theorem 2 is trivially true. Note that conditions (iii) and (iv) imply that if $r \geqslant 2$ then $r \geqslant 3$ and $r$ is odd. As mentioned earlier, we define $p_{\varepsilon}$ to be the probability that two points $x, y$ (chosen at random from $[0,1]$ according to the uniform distribution and subject to the condition $|x-y|<\varepsilon)$ tend to different attractors. That is, let $I_{\varepsilon}(x)=[x-\varepsilon, x+\varepsilon]$ and set

$$
p_{\varepsilon}(x)=m\left(\left\{y \in I_{\varepsilon}(x) \cap[0,1]: \lim _{n} f^{n}(x) \neq \lim _{n} f^{n}(y)\right\}\right) / m\left(I_{\varepsilon}(x) \cap[0,1]\right)
$$

and then set $p_{\varepsilon}=\int_{0}^{1} p_{\varepsilon}(x) d x$. Then we have

THEOREM 2.

$$
\lim _{\varepsilon \rightarrow 0} \frac{\log p_{\varepsilon}}{\log \varepsilon}=1-\alpha,
$$

where $\alpha=\operatorname{cap}(\Lambda)=\operatorname{HD}(\Lambda)$.

Remark. Theorem 2 is true for $q_{\varepsilon}$ also. Having established the equality of Hausdorff and Capacity dimensions, one proceeds as follows. Cover $\Lambda$ by $N(\varepsilon)$ intervals of length $\varepsilon$. Every point in the union of these intervals is within $\varepsilon / 2$ of $\Lambda$. Although the intervals are not necessarily disjoint, no point in their union is contained in more than two of the intervals since $N(\varepsilon)$ was the minimum number of intervals required to cover $\Lambda$. Hence, $q_{\varepsilon / 2} \geqslant \frac{1}{2} N(\varepsilon) \varepsilon$. If we replace each of the intervals in the cover with intervals having the same midpoint but twice the length, every point within $\varepsilon$ of $\Lambda$ is contained in the union of these intervals and so $q_{\varepsilon} \leqslant N(\varepsilon) \varepsilon 2$. Taken together, these inequalities give

$$
\lim _{\varepsilon \rightarrow 0^{+}} \frac{\log q_{\varepsilon}}{\log \varepsilon}=1-\operatorname{cap}(\Lambda) .
$$

Proof of Theorem 2. Let

and

$$
A(\varepsilon)=\{(x, y) \in[0,1] \times[0,1]:|x-y|<\varepsilon\}
$$

$$
D(\varepsilon)=\left\{(x, y) \in A(\varepsilon): \lim _{n} f^{n}(x) \neq \lim _{n} f^{n}(y)\right\} .
$$

Then, denoting Lebesgue measure on $[0,1]^{2}$ by $m$, we have that $p_{\varepsilon}$ has the same behavior (as $\varepsilon \rightarrow 0$ ) as $m(D(\varepsilon)) / m(A(\varepsilon))$. Note that $m(A(\varepsilon))=2 \varepsilon-\varepsilon^{2}$. Thus, to prove the theorem, it is enough to show that $\lim _{\varepsilon \rightarrow 0} \log m D(\varepsilon) / \log \varepsilon=2-\alpha$.

We define a mapping $F:[0,1]^{2} \rightarrow \mathbf{R}^{2}$ by $F(x, y)=(f(x), f(y))$. It is immediate that $\left|\operatorname{det} D F^{n}(x, y) / \operatorname{det} D F^{n}(w, z)\right| \leqslant K^{2}$ whenever $(x, y)$ and $(x, z)$ belong to the same set $C_{j}^{n} \times C_{j}^{n}$. Also, note that for each $n$ and $j$ we have that

$$
D\left(K^{-1} \varepsilon\left|C_{j}^{n}\right|^{-1}\right) \subset F^{n}\left(D(\varepsilon) \cap\left(C_{j}^{n} \times C_{j}^{n}\right)\right) \subset D\left(K \varepsilon\left|C_{j}^{n}\right|^{-1}\right)
$$


for some $k$. Our goal is to derive a scaling law for $D(\varepsilon)$ analogous to equation (2). In order to do this we will estimate $m(D(\varepsilon))$ in terms of $m\left(D(\varepsilon) \cap\left(\cup_{j} C_{j}^{n} \times C_{j}^{n}\right)\right)$, and then apply (5).

Begin by considering $D(\varepsilon)$ with

$$
\varepsilon \ll \min \left\{\min _{j \neq k} \operatorname{dist}\left(C_{j}^{n+2}, C_{k}^{n+2}\right), \min _{j}\left|C_{j}^{n}\right|\right\}
$$

Then $(x, y) \in D(\varepsilon)$ implies that one of $x, y$ lies in some $C_{j}^{n}$. In this case $(x, y)$ must lie in the region $*_{\varepsilon} C_{j}^{n}$ shown in Figure 2.

Claim. There is a subset of $\bigcup_{j} C_{j}^{n} \times C_{j}^{n}$ mapped in a 1-1 manner onto $\cup_{j} A_{j}^{n} \cup B_{j}^{n}$ by $F^{2}$. To establish the claim it is enough to show the following: If $C_{j}^{n}=[a, b]$ then at least one of the points in $f^{-2}(a)$ (and at least one of the points in $f^{-2}(b)$ ) lie in the interior of some $C_{l}^{n}$. Such a point is an endpoint of some $C_{k}^{n+2}$. The corresponding set $A_{k}^{n+2}$ (or $B_{k}^{n+2}$ ) determined by $*_{\varepsilon} C_{k}^{n+2}$ is contained in some $C_{l}^{n}$ and is mapped onto $A_{j}^{n}$ (or $B_{j}^{n}$ ) by $F^{2}$.

Proof of Claim. Suppose $C_{j}^{n}=[a, b]$ and that all of the points $f^{-2}(a)$ are endpoints of sets $C_{l}^{n}$. Apply $f^{n-1}$. We obtain a set $C_{j}^{1}=\left[a^{\prime}, b^{\prime}\right]$ such that all the points $f^{-2}\left(a^{\prime}\right)$ are endpoints of other intervals $C_{k}^{1}$. But there are $r$ intervals $C_{k}^{1}$ and $r^{2}$ points in $f^{-2}(a)$. Since $r^{2}>2 r$ (when $r>2$, as we have assumed), this is a contradiction.

Thus $D(\varepsilon) \cap\left(\cup_{j} A_{j}^{n} \cup B_{j}^{n}\right)$ is contained in the image (under $F^{2}$ ) of a subset of $D(\varepsilon) \cap\left(\cup_{j} C_{j}^{n} \times C_{j}^{n}\right)$. Since $F^{2}$ expands areas by at most a factor $B^{\prime} \geqslant 1$, we have shown that (set $B=B^{\prime}+1$ )

$$
m(D(\varepsilon)) \leqslant B m\left(\bigcup_{j} D(\varepsilon) \cap C_{j}^{n} \times C_{j}^{n}\right)
$$

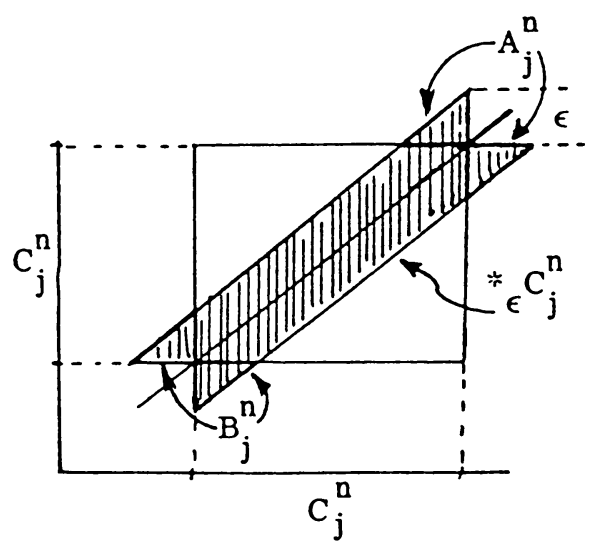

Figure 2 
We can now apply (5), sum over $j$ and obtain

$$
K^{-2} \sum_{j} m\left(D\left(\varepsilon K^{-1}\left|C_{j}^{n}\right|^{-1}\right)\right)\left|C_{j}^{n}\right|^{2} \leqslant m\left(D_{\varepsilon}\right) \leqslant B K^{2} \sum_{j} m\left(D\left(K \varepsilon\left|C_{j}^{n}\right|^{-1}\right)\right)\left|C_{j}^{n}\right|^{2}
$$

Finally, suppose $2-d>\operatorname{cap}(\Lambda)$ and write $m\left(D_{\varepsilon}\right)=\varepsilon^{d} \phi(\varepsilon)$. Select $n$ so large that $\sum_{j}\left|C_{j}^{n}\right|^{2-d}<\left(B K^{2}\right)^{-1}$. When $\varepsilon$ is sufficiently small, equation (6) yields

$$
\phi(\varepsilon) \leqslant B K^{2} \sum_{j}\left|C_{j}^{n}\right|^{2-d} \phi\left(K \varepsilon\left|C_{j}^{n}\right|^{-1}\right)
$$

which implies that $\phi$ is bounded as $\varepsilon \rightarrow 0$. Hence

$$
\lim _{\varepsilon \rightarrow 0} \frac{\log m D(\varepsilon)}{\log \varepsilon}=\lim _{\varepsilon \rightarrow 0} d+\frac{\log \phi(\varepsilon)}{\log \varepsilon} \geqslant d
$$

for every $d<2-\operatorname{cap}(\Lambda)$.

Then let $2-d<\operatorname{cap}(\Lambda)$ and, selecting $n$ so large that $\sum_{j}\left|C_{j}^{n}\right|^{2-d}>K^{2}$, write $m\left(D_{\varepsilon}\right)=\varepsilon^{d} \phi(\varepsilon)$. The left-hand inequality in (6) then yields

$$
\phi(\varepsilon) \geqslant \sum_{j} K^{2}\left|C_{j}^{n}\right|^{2-d} \phi\left(K^{-1} \varepsilon\left|C_{j}^{n}\right|^{-1}\right)
$$

This means $\phi(\varepsilon)$ is bounded away from zero and so

$$
\varlimsup_{\varepsilon \rightarrow 0} \frac{\log m D(\varepsilon)}{\log \varepsilon}=d+\varlimsup_{\varepsilon \rightarrow 0} \frac{\log \phi(\varepsilon)}{\varepsilon} \leqslant d
$$

for every $d>2-\operatorname{cap}(\Lambda)$.

REMARK. The quantity $p_{\varepsilon}$ was interpreted as the probability of making an error when predicting the long-term behavior of the system in the presence of a uniform error (of size $\varepsilon$ ) in determining the initial conditions of the system. The assumption that the errors are uniform is not, however, necessary. Let $\mu_{\varepsilon}$ be any probability measure on $[-\varepsilon, \varepsilon]$ which is equivalent to normalized Lebesgue measure on $[-\varepsilon, \varepsilon]$. (That is, both $\left(d \mu_{\varepsilon} / d x\right) / 2 \varepsilon$ and $2 \varepsilon /\left(d \mu_{\varepsilon} / d x\right)$ are bounded independent of $\varepsilon$.)

We suppose that $\mu_{\varepsilon}$ describes the errors in observing the states of the system, and define a quantity $\bar{p}_{\varepsilon}$ analogous to $p_{\varepsilon}$. Set

$$
\overline{p_{\varepsilon}}(x)=\mu_{\varepsilon}\left\{y \in[-\varepsilon, \varepsilon]: x+y \in[0,1] \text { and } \lim _{n} f^{n}(x) \neq f^{n}(x+y)\right\}
$$

and $\overline{p_{\varepsilon}}=\int \overline{p_{\varepsilon}}(x) d x$. Then $\overline{p_{\varepsilon}}$ has the same behavior (as $\varepsilon \rightarrow 0$ ) as $p_{\varepsilon}$. This is because the quantities $m\left(D(\varepsilon) \cap C_{j}^{n} \times C_{j}^{n}\right)$ in the proof of Theorem 2 change by at most a bounded factor when Lebesgue measure is replaced by $\mu_{\varepsilon}$.

\section{Two-dimensional examples.}

A. Rational maps. We begin with an explicit example: $f(z)=z^{2}+c$ with $c \in \mathbf{C}$ of sufficiently small modulus. Considered as a map of the Riemann sphere $\mathbf{C} \cup\{\infty\}$, the map $f$ has two attracting fixed points, one near 0 and the other at $\infty$. The common boundary of the basins of these attractors is the Julia set of $f$, frequently denoted $J(f)$. 

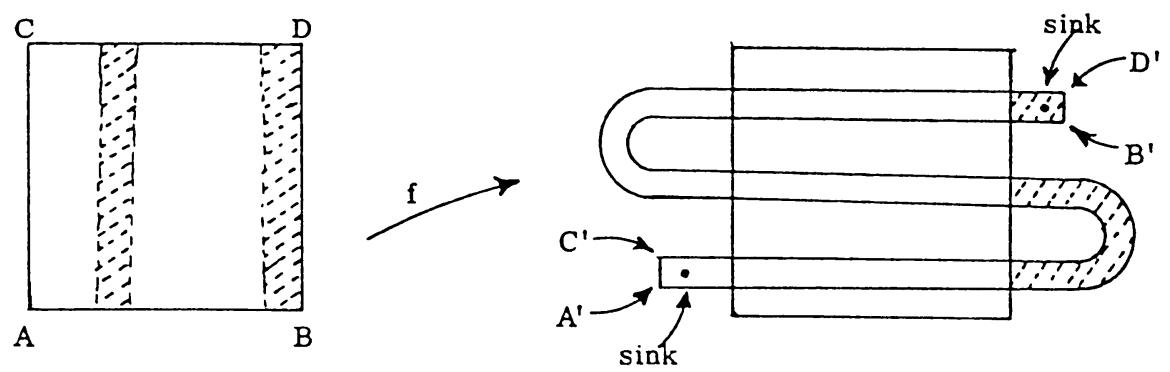

This illustrates the action of the "horseshoe" diffeomorphism on the square $A B C D$. The image of the square (relative to itself) is shown on the right. The shaded region indicates part of the basin of attraction of one of the sinks.

\section{FIGURE 3}

More generally, we suppose that $f$ is a rational map of the sphere $\mathbf{C} \cup\{\infty\}$ which has two attracting fixed points (say 0 and $\infty$ ). In addition, suppose that the forward orbit of each critical point of $f$ tends to one of the attractors. Then (see [3]) the common boundary of the basins of the attractors of $f$ is the set $J(f)$ and some power of $f$, say $f^{r}$, is uniformly expanding on a neighborhood of $J(f)$.

Let $N$ be a neigborhood of $J(f)$ on which $f^{r}$ is uniformly expanding. (That is, $\left|(d / d z) f^{r}(z)\right| \geqslant \gamma>1$ for $z \in N$.) Denote by $p_{\varepsilon}$ the probability that $x, y$ chosen at random from $N$ (according to the uniform distribution and subject only to $|x-y|$ $<\varepsilon$ ) tend to different attractors under the action of $f$. Then, corresponding to Theorems 1 and 2, we have

$$
\operatorname{HD}(J(f))=\operatorname{cap}(J(f))
$$

and

$$
\lim _{\varepsilon \rightarrow 0^{+}} \frac{\log p_{\varepsilon}}{\log \varepsilon}=2-\operatorname{HD}(J(f)) .
$$

The proofs of (A1) and (A2) are almost identical with the proofs of the corresponding theorems. The one difference is that, instead of using the sets $\left\{C_{j}^{n}\right\}$, we use sets of the form

$$
\left\{z: f^{j}(z) \in A_{k_{j}}, j=0,1, \ldots n-1, k_{j} \in\{1, \ldots, m\}\right\}
$$

where $\left\{A_{1}, \ldots, A_{m}\right\}$ is some cover of $J(f)$ by open sets.

It should be noted that this (A2) provides a method for computing the Hausdorff dimension of some Julia sets which is particularly well-suited to the use of parallel or vector processors.

B. Horseshoes. Consider a horseshoe diffeomorphism which acts on a disk in the plane as indicated in Figure 3.

There are two attracting fixed points for $f$. Trajectories starting at Lebesgue almost every point tend to one of these attractors under the action of $f$. The boundary of the basins of the attractors is the stable manifold $W^{s}$ of the horseshoe. If we assume that $f$ restricted to $f^{-1}(R)$ is an affine transformation which preserves 
vertical lines (so that $W^{s}$ is the product of a Cantor set and an interval) we obtain, as an application of Theorem 2, that

(B1)

$$
\lim _{\varepsilon \rightarrow 0} \frac{\log p_{\varepsilon}}{\log \varepsilon}=2-\operatorname{HD}\left(W^{s}\right) .
$$

\section{REFERENCES}

1. C. Grebogi, S. McDonald, E. Ott and J. Yorke. Final state sensitivity: an obstruction to predictability, Phys. Lett. 99A (1983), 415-418.

2. D. Farmer, E. Ott and J. Yorke. The dimension of chaotic attractors, Physica 7D (1983), 153-180.

3. P. Blanchard, Complex analytic dynamics on the Riemann sphere, Preprint.

Institute for Mathematics and Its Applications, University of Minnesota, MinNeApolis, MinNESOTA 55455

Current address: Department of Mathematical Sciences, University of Cincinnati, Cincinnati, Ohio 45221 